J. theor. Biol. (2002) 218, 97-109

doi:10.1006/yjtbi.3054, available online at http://www.idealibrary.com on IDE $\mathbf{A}^{\circledR}$

\title{
Red Queen Dynamics of Protein Translation
}

\author{
David C. Krakauer* $†$ and Vincent A. A. Jansen $\uparrow$ \\ $\dagger$ Santa Fe Institute, Hyde Park Road, Santa Fe, NM 87501, U.S.A. and $\ddagger$ School of Biological Sciences, \\ Royal Holloway, University of London, Egham, Surrey TW20 0EX, U.K.
}

(Received on 23 July 2001, Accepted in revised form on 8 April 2002)

\begin{abstract}
We explore adaptive theories for the diversity of translational binding based on the genetic code viewed as a primitive mechanism of resistance. Modifying the set of codons bound by tRNA anticodon molecules or changing the specificity of binding, reduces the replication rate of translational parasites such as viruses. Increased translational efficiency of the parasite requires a high degree of specificity of host tRNAs for the parasite codons. This suggests that the genetic code might serve as the first line of defense against infection. We construct a red queen theory for translational diversity: a theory in which host-translational strategiesas defined by the degree of redundancy (a single anticodon binding many codons for a single amino acid) or degeneracy (many anticodons binding many codons for a single amino acid) - are constantly shifting through time to evade parasitism but where neither parasite nor host gain a systematic advantage.
\end{abstract}

(C) 2002 Elsevier Science Ltd. All rights reserved.

\section{The Code and Codon Assignment}

The mechanisms of protein synthesis via translation remain largely invariant across taxa. Each amino acid destined to become a component of a protein is associated with one or more codons of three nucleotides of RNA. Each codon becomes associated with an amino acid through an adaptor molecule carrying an amino acid, the transfer RNA (tRNA). The tRNA binds to the codon with a complementary anticodon according to the Watson-Crick base-pairing rules. These describe how the purines adenine and guanine bind with the pyrimidines thymine, cytosine and uracil. The mapping from codon to amino acid is many to one and this gives rise to synonym redundancy in the genetic code. One mechanism for ensuring that each of the 64

\footnotetext{
*Corresponding author.

E-mail address: krakauer@santafe.edu (D.C. Krakauer), vincent.jansen@rhul.ac.uk (V.A.A. Jansen).
}

possible codons is associated with at least one of the 20 possible amino acids is through "wobble" of the first or third nucleotide base of the codon. This arises in part as the first and third sites of the anticodon bind more weakly than the second. As a consequence, in many triplets the third base of a codon is by and large "silent", reducing the specificity of the anticodon and thereby expanding the set of acceptable codons.

Adaptive explanations for the structure of the genetic code tend to focus on the non-random assignment of amino acids to codons. These theories address why particular codons encode a given amino acid and why these associations remain conserved across distantly related taxa. Mutational load hypotheses observe that the 20 sense codon phenotypes (encoding 20 amino acids), are assigned to 61 codons so as to minimize the phenotypic expression of mutations to the DNA at the amino acid sequence (Haig \& Hurst, 1991; Freeland \& Hurst, 1998). 
The code is interpreted as error buffering, ensuring that biochemically similar amino acids are encoded by sets of similar codons. A good example of this correspondence is that amino acids with a $\mathrm{U}$ at the second codon position are all hydrophobic whereas those with an A are all hydrophilic. These adaptive theories, can help to explain how the otherwise arbitrary codon assignments in the genetic code show so little variability across distantly related species. In this paper, we are not considering the adaptive benefit of assignment. We consider another feature of the genetic code, which shows far greater variability: the diversity of translational binding. Whereas adaptive theories for assignment rules seek to explain uniform features of the genetic code, we seek to explain using adaptive arguments, diverse features of the genetic anticode - the mapping between codon and anticodon or anticodon and amino acid.

\section{Translational Parasitism}

In this paper, we explore the result of assuming that the code evolved and persists in an environment in which there is a constant risk of translational parasitism. This takes the form of short sequences of RNA or DNA which make use of a host's protein-synthesis machinery. A contemporary example is a virus, an obligate parasite, incapable of self-replication without host factors. Increased resistance against infection can be achieved in two ways: by (1) biasing the anticodons of the host tRNA pool away from the parasite codon set through reduced anticodon-codon binding specificity, or (2) modifying the copy number of a tRNA anticodon required by the parasite. These strategies can be effective as the translation of highly expressed parasite genes requires large numbers of complementary anticodons in the host (Bennetzen \& Hall, 1982; Kurland, 1991; Lammertyn et al., 1996). Modifying anticodons and tRNA availabilities restrict virus genomes access to host protein-synthesis machinery.

\section{ANTICODON REDUNDANCY AND DEGENERACY}

Anticodon redundancy will be taken to refer to cases where identical elements (anticodons) can process alternative inputs (different codons) to produce a single outcome (translation into an amino acid). Whereas anticodon degeneracy involves non-identical elements (different anticodons) processing alternative inputs (different codons) to produce a single outcome (translation into an amino acid). These two strategies are illustrated in Fig. 1. Within the standard code, the total number of tRNAs can vary theoretically between 22 , in which case each anticodon recognizes on average three codons, to 61 in which case each anticodon binds strictly to its complementary codon (setting aside the three stop codons). This leads to two or more tRNAs carrying the same amino acid (isoaccepting tRNAs). Using the above terminology, employing a large number of specific anticodons (61 for example) to encode 20 amino acids is a strategy of degeneracy, as non-identical anticodons must map onto a common set of amino acids. By employing a smaller number of anticodons than codons, the system becomes redundant, as the same anticodon is required to bind to more than one codon.

Anticodon redundancy arises through modification of the standard Watson-Crick basepairing rules, whereas degenerate anticodons arise by ensuring that the rules are interpreted strictly. Assuming complete degeneracy 61 tRNAs are required to bind all 20 amino acids. For both redundancy and degeneracy, each of the tRNAs is charged with one amino acid by one of 20 tRNA synthetase enzymes and thereby both lead to synonym redundancy in the code.

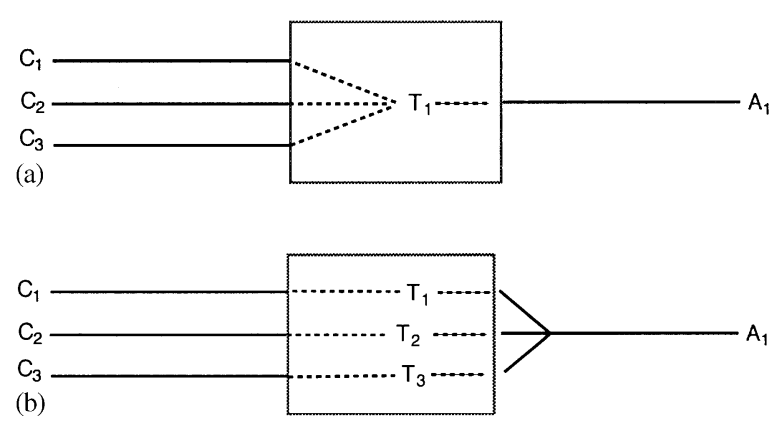

FIG. 1. Anticodon redundancy (1) and anticodon degeneracy (b). Anticodon redundancy requires that a specific anticodon $\left(T_{i}\right)$ recognizes several different codons $\left(C_{i}\right)$ and maps these onto a single amino acid $\left(A_{1}\right)$. Degeneracy involves anticodons ( $\mathrm{Ti})$ binding to codons $(\mathrm{Ci})$ in which each of the anticodons is associated with the same amino acid. 
A redundant strategy allows genomes to carry fewer tRNA molecules and acts as a simple mechanism for error buffering by not distinguishing among similar codons. However, redundancy involves a potential cost in terms of reduced binding specificity and more frequent mismatch errors (Knight et al., 1999).

Degeneracy provides greater specificity of binding, reducing translation errors by reducing the incidence of near-cognate codon readings. It also has the benefit of greater evolutionary flexibility as the number of elements available for potential modification is increased. With a degenerate strategy, the set of bound codons can be modified by simply expanding or contracting the set of anticodons. However, a degenerate strategy requires more tRNAs and an attendant increase in the genome size. Large genomes can impose a fitness cost both in terms of reduced replication rates and increased mutational loads.

Anticodon redundancy and anticodon degeneracy are of interest as they influence the fidelity of translation, the rate of replication, and code modifiability.

The degree of redundancy at one extreme and degeneracy at the other spans the theoretical range. Metazoan mitochondria decode all codons with around 22 different tRNAs. In order to do so, mitochondria have evolved special redundant mechanisms of translation and have zero degeneracy. Each mitochondrial tRNA is used for a set of two or four redundant codons. Those tRNAs interacting with a four-member set, employ a $U$ at the $5^{\prime}$ terminus of the anticodon able to interact with any of the four bases of the codon. In contrast, Homo sapiens carry around 51 unique tRNAs; $C$. elegans around 47; Drosophila melanogaster 44; Saccharomyces cerevisiae 41; Haemophilus influenzae 36; Helicobacter pylori 33; and Borrelia burgdorferi 29. In Fig. 2, we have plotted the number of different tRNA genes as a function of genome size for a number of procaryotic and eucaryotic species. One finds that genome size is a strong determinant or translational strategy. Smaller genomes favor redundancy, whereas larger genomes favor degeneracy. This observation relates to Kurland's seminal work on the econimization principle, wherein he proposes that genome size exerts a very strong selection

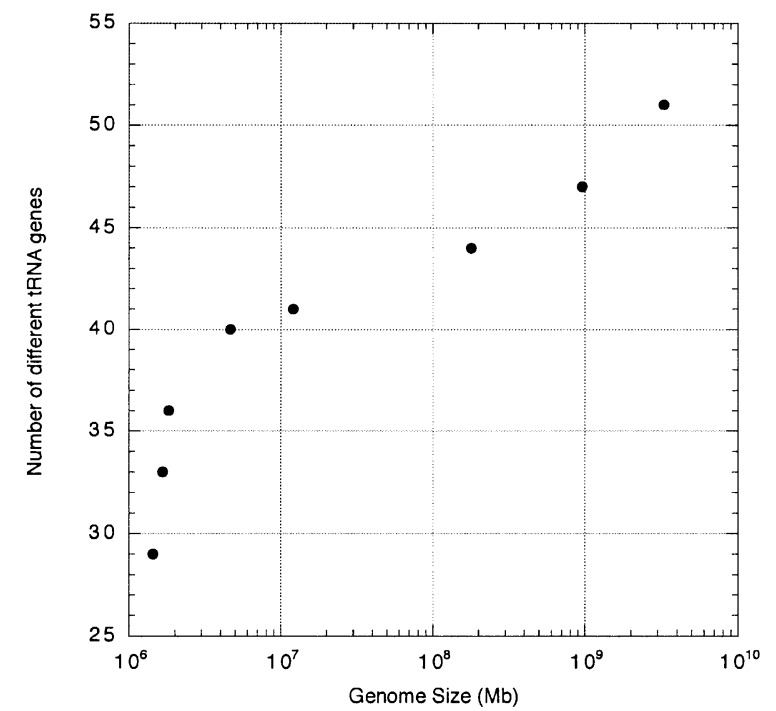

FIG. 2. The relationship between genome size and tRNA gene numbers for three eucaryotic and five procaryotic species. One observes a strong influence of genome size on the degree of anticodon redundancy and degeneracy. Small genomes tend towards redundancy, whereas large genomes favor degeneracy.

pressure on redundancy and on the modifiability of the genetic code (Anderson \& Kurland, 1998).

A third source of redundancy in the code relates to the number of tRNA gene copies associated with each anticodon. Thus, for a given amino acid there can be one or more tRNA gene copies, the numbers of which influence the efficiency of translation. Increasing the number of duplicates of a given tRNA has been shown to significantly amplify the rate of translation of highly expressed genes (Bulmer, 1991; Dong et al., 1995; Sharp et al., 1993).

To explore how translational parasitism might influence translational strategies, we have developed a number of models in which parasites and their hosts vary the degree of effective synonym redundancy in their genomes (how many synonymous codons are employed), and in which hosts can vary the specificity and number of their tRNA molecules (degeneracy). Such models have obvious parallels with the "red queen" hypothesis for the evolution of sex (Hamilton et al., 1990). In this hypothesis diversity among genes is associated with greater resistance to infection. Here, the hypothesis is extended, to encompass the structure of the translational apparatus. The red queen metaphor describes 
situations in which improvements in one species are matched by commensurate improvements in a competitor (Van Valen, 1973). Applied to protein synthesis, the net result is that whereas translational strategies change, mean fitness remains constant. In this paper, we explore the coevolution of host translation and parasite codon usage in order to understand: (1) what the consequences of different degrees of redundancy and degeneracy are for virus codon usage and replication, (2) how changes in translation strategies at the cellular level are influenced by virus replication at the population level, (3) review the experimental data relating to molecular mechanisms that might modify host translation, and (4) provide evidence for shifting parasite codon usage and its fitness effects.

\section{INFECTION, TRANSLATION, REDUNDANCY, AND DEGENERACY}

To quantify redundancy and degeneracy, and to explore the consequences of these factors on the translation of a parasite's genome, we develop a model for the translation process of RNA into proteins. For expositional reasons, we start by considering the translation of a pair of synonymous codons by means of a pair of anticodons into a single amino acid. A generalized model for translation of larger sets of codons is presented in Appendix A.

Consider the translation of a pair of codons into a single amino acid. We assume that the fraction of anticodons of type $1, v_{1}$, is given by $\alpha$ and consequently the fraction of host anticodons of type $2, v_{2}$, by $1-\alpha$. These anticodons can be used by the parasites to translate its codons. We assume that the parasite can potentially use two different codons, which occur with fractions $c_{1}=$ $\gamma$ and $c_{2}=1-\gamma$. Both codons can in principle bind to both anticodons but do so with different affinities. We assume that the affinity of codon $i$ for anticodon $j$ is $d(i, j \in\{1,2\}, i \neq j)$ and the affinity of anticodon $i$ for codon $j$ is $1-d$ $(i, j \varepsilon\{1,2\}, i=j)$. If $d$ is small a codon and anticodon with the same label will pair with a high probability, whereas when $d$ is close to 1 the reverse is true. As the codon abundance of the parasite can differ from that of the host, the host can to a certain extent control the replication of parasites within its cells. We will develop a simple mechanistic description of the translation process to infer the consequences of redundancy and degeneracy on the translation rate of parasite genomes.

The total rate of binding to codon 1 is given by $g_{1}=v_{1}(1-d)+v_{2} d=\alpha(1-d)+(1-\alpha) d$ and the rate of binding to codon 2 is $g_{2}=$ $v_{1} d+v_{2}(1-d)=1-g_{1}$. The average time it takes for codon $i$ to be matched is given by $f_{i}=$ $1 / g_{i}$. The total translation time is given by the sum of the time it takes to initiate the translation process, $\varepsilon$ (binding of the message to the ribosome), and the total time to match all codons

$$
\tau=\varepsilon+c_{1} f_{1}+c_{2} f_{2} .
$$

Assuming that the extension of the protein is the rate limiting step, and not the removal of improperly bound anticodons, the rate of translation is given by $q=1 / \tau$. Given these assumptions, the rate of translation of a single amino acid of a parasite $l$ in a host $k$, is given by

$$
\begin{aligned}
& q(d, \gamma) \\
& =\left(\frac{1-\gamma}{1-(d+\alpha-2 d \alpha)}+\frac{\gamma}{d+\alpha-2 d \alpha}+\varepsilon\right)^{-1} .
\end{aligned}
$$

The parasite is able to modify only the value of $\gamma$, its codon usage, specifying the relative frequency of synonymous codons. We can ask what value of $\gamma$ maximizes the rate of translation provided fixed host anticodon abundances $v_{i}$ and binding affinities. One finds that this depends on the binding rates

$$
\begin{array}{ll}
g_{1}>g_{2} & \text { then } \gamma=1, \\
g_{2}<g_{1} & \text { then } \gamma=0 .
\end{array}
$$

This tells us that small biases in the host affinity for codons, results in the exclusive use of the favored codons by the parasite. The same reasoning should hold for the translation of host messages - all else being equal the host can optimize its translation process by only using those codons that give rise to the highest translation rate. Alternatively, the host can adjust the affinity matrix or anticodon abundance to optimize translation. However, codon 
usage also determines the error rate during transcription into mRNA of its nuclear DNA. A more extensive discussion of translation efficiency is provided Bulmer (1991) and Xia (1998).

These assumptions serve as a simple mechanistic background for the dynamical models that follow. We place the translational kinetics into the larger context of parasite-host population dynamics. This allows us to explore the ways in which population level selection feeds back to modify parasite codon usage and host-translational strategies.

\section{POPULATION DYNAMICS}

The abundance of hosts is given by $x$ and the abundance of parasites by $y$. We assume that parasites infect hosts with an efficiency proportional to translation rates. We also assume that host-replication rate does not depend on the translation rate of its own genes. Of course, hostreplication rates must at a certain point depend on translation rates: when translation rate approaches zero, hosts cease to exist! However, for all but a small number of highly expressed genes, variation in translation rate is not critical for host-protein production. This is in contrast to viruses in which replication and translation are tightly coupled in the life cycle. We can therefore write down differential equations to capture the population dynamics of parasites and hosts as

$$
\begin{aligned}
& \dot{x}=x(r-y q(d, \gamma)), \\
& \dot{y}=y(x q(d, \gamma)-\delta) .
\end{aligned}
$$

Hosts replicate at a rate $x r$ and experience a parasite-induced mortality proportional to the replication rate of the parasite and the parasite abundance, $y q(d, \gamma)$. The value $q$ is a function of $d$ and $\gamma$. The parasite replicates at a rate proportional to its translation within the host cells and the total host abundance $x q((d, \gamma)-\delta)$ and experiences mortality at a rate $x \delta$. We select a very simple dynamics whereby hosts and parasites are completely antagonistic and parasite transmission is horizontal. As a result, the host and the parasite have no common interest.
A more complete formulation takes account of parasites interested in vertical transmission, in which case host and parasite share a common good [see van Baalen \& Jansen (2001) for detailed discussion].

The population dynamics of this model are well known; the densities show neutrally stable oscillations around the equilibrium. The mean densities of hosts and parasites over these oscillations equal the respective equilibrium densities. The equilibrium abundances of the parasites and hosts are given by

$$
\hat{x}=\delta / q(d, \gamma) \text { and } \hat{y}=r / q(d, \gamma) .
$$

Thus, any increase in the rate of translation leads to a concomitant reduction in the abundance of both parasite and host. A reduction in the rate of translation of host genes, leads to a reduction in the abundance of parasites without any impact on the host abundance. This is not surprising as we assume that other factors influence hostreplication rates. Parasites influence the host primarily through increased mortality. Given our assumptions, we hypothesize that over the course of evolution, translation within the host should become less and less efficient.

\section{EVOLUTIONARY DYNAMICS OF SINGLE PARASITE AND HOST}

We now embed our translation model within a population dynamics model of hosts and parasites. In this way, we can derive microscopically the evolutionary dynamics of redundancy. We do so considering the fate of mutant hosts with different redundancies. For simplicity, we assume that a change in redundancy is achieved through a change in the parameter $d$. This single parameter allows us to vary redundancy between 0 when $d=1$ or 0 and its maximum when $d=$ 0.5 . We do not vary the translation error rate or the genome replication rate as a function of the level of redundancy or degeneracy in this paper. They consequently exert similar influences on the population dynamics. Here, we seek to show how feedback from population dynamics can influence the genetic anticode. In [Krakauer, 2002], the differences between redundancy and degeneracy are explored more thoroughly. We will denote the mutant parameter with 
a superscripted star, hence the mutant affinity is given by $d^{*}$. Similarly, we will consider mutant parasites that have a different codon usage, $\gamma^{*}$. The invasion rate of such a mutant when it is rare, relative to the other resident strains, is a measure of its fitness (Metz et al., 1992). If a mutant host is rare it will predominantly be infected by a resident parasite. Similarly, mutant parasites will predominantly infect resident hosts. The dynamics of the resident hosts and parasites are approximated by system (2) above as long as the mutants are rare, and the average densities are given by $\hat{x}$ and $\hat{y}$. The dynamics of rare mutant hosts, $x^{*}$ and parasites $y^{*}$ is given by

$$
\begin{aligned}
& \dot{x}^{*}=x^{*}\left(r-y q\left(d^{*}, \gamma\right)\right), \\
& \dot{y}^{*}=y^{*}\left(x q\left(d, \gamma^{*}\right)-\delta\right) .
\end{aligned}
$$

The average per capita growth rate of a mutant host when rare is $r-\hat{y} q\left(d^{*}, \gamma\right)=r(1-$ $\left.\left(q\left(d^{*}, \gamma\right) / q(d, \gamma)\right)\right)$ and the average per capita growth rate of a mutant parasite when rare, $\hat{x} q\left(d, \gamma^{*}\right)-\delta=\delta\left(\left(q\left(d, \gamma^{*}\right) / q(d, \gamma)\right)-1\right)$. These expressions show that the mutant parasite can always invade if its translation rate $q\left(d, \gamma^{*}\right)$ is larger than that of the resident parasite, whereas the mutant host can invade if it has a lower value of $q\left(d^{*}, \gamma\right)$ than the resident host. These expressions also show that the host that leads to the highest parasite density cannot be replaced by any other hosts. The parasite that induces the lowest host density cannot be replaced by other parasites. Host evolution thus maximizes parasite density while parasite evolution minimizes host density. This is also known as the pessimization principle (Mylius \& Diekmann, 1995).

In creating these worst of possible worlds, the hosts are selected to minimize $q$ whereas the parasites are selected to maximize it. Host and parasites thus have opposing evolutionary interests. This raises the question as to what translation rate will be finally established and the nature of the evolutionary dynamics of codon usage and redundancy.

To reconstruct the evolutionary dynamics of $d$ and $\gamma$, we will derive the evolutionary dynamics of a trait, following Dieckmann \& Law (1996) and Lande (1979). We first consider the fitness of a host with a marginally different affinity $d^{*}=$ $d+\varepsilon$. The fitness of this mutant is approximately $-(\varepsilon r / q(d, \gamma)) \partial q\left(d^{*}, \gamma\right) /\left.\partial d^{*}\right|_{d^{*}=d}=-\varepsilon \hat{y} \partial q / \partial d$. The time it takes for a mutant to replace the resident is proportional to $-\hat{y} \partial q / \partial d$, a quantity also known as the selection differential. The probability for such a mutant to appear per unit of time is proportional to the total number of individuals, $\hat{x}$, multiplied by a constant to incorporate the mutation rate and average mutational change $\mu_{d}$. The total change in the value of $d$ over time is proportional therefore to $-\mu_{d} \hat{x} \hat{y} \partial q / \partial d$. For $\gamma$, a similar argument can be applied. This yields the following system of differential equations:

$$
\begin{gathered}
\dot{d}=-\mu_{d} \hat{x} \hat{y} \frac{\partial q}{\partial d}, \\
\dot{\gamma}=\mu_{\gamma} \hat{x} \hat{y} \frac{\partial q}{\partial \gamma} .
\end{gathered}
$$

The parameter $\mu_{\gamma}$ represents the mutation rates of $\gamma$. To restrict the evolutionary dynamics to the unit square these parameters take the value zero if mutation is outside the unit interval.

To analyze the evolutionary dynamics, we locate the equilibrium of this system. The isocline for $\gamma$ is given by $d=1 / 2$, the isocline for $d$ by $\gamma=(d+\alpha-2 d \alpha)^{2}(1-2(d+\alpha-$ $2 d \alpha)(1-(d+\alpha-2 d \alpha)))^{-1}$. The isoclines intersect at $d=1 / 2, \gamma=1 / 2$ at which point the equilibrium of this dynamical system can be found. If the affinity of the codon-anticodon bond is the same for both pairs and if the parasite uses both codons this state cannot be altered by coevolution. Note that there is maximum redundancy for these parameter values.

This does not mean that the evolutionary dynamics proceed to this equilibrium. Simulations show that the dynamics spiral away from this point (Fig. 3). The host and the parasites continuously alter their codon usage and redundancy to make life worse for their competitors. In doing so they modify the translation rate in opposite directions. This results in a never ending arms race in which both partners have to keep running in order to stand still: the evolutionary dynamics of the red queen. If the 


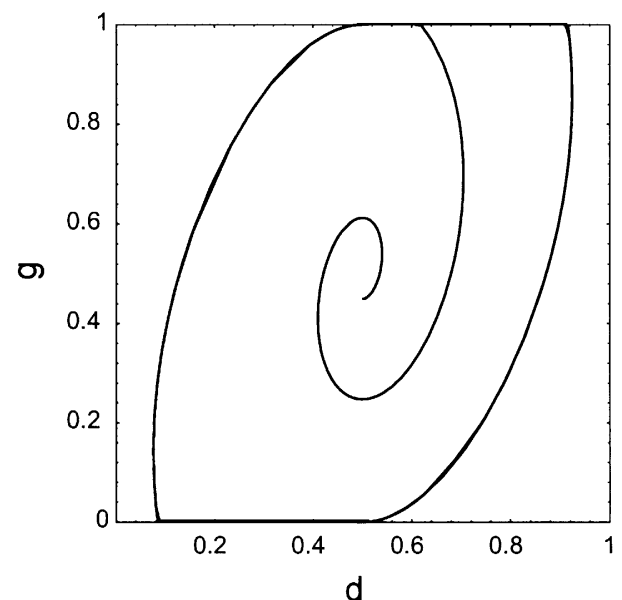

FIG. 3. The oscillatory (red queen) dynamics of monomorphic host and parasite populations. The dynamics are depicted as a phase portrait for the parameters $d$ and $\gamma$. One observes cycles in the preferred parameter values, with an increase in redundancy, there is a concomitant decrease in parasite codon usage and vice versa. Parameter values are: $\varepsilon=1, \alpha=\beta=0.25, r=\delta=0.1 \mu_{d}=0.25, \mu_{\gamma}=1$.

parasite's codon usage should remain constant, the host would modify its codon usage such that it employed a single, non-matching codon and redundancy would be minimized. However, the cyclic evolutionary dynamics cause the redundancy to fluctuate between extreme values (Fig. 4).

\section{EVOLUTIONARY DYNAMICS OF TWO PAIRS OF HOST AND PARASITES}

In the previous section it was tacitly assumed that the host and parasite populations are monomorphic. There is no reason a fortioriori why this should be the case. In fact, more than one pair of hosts and parasites can coexist. The simplest example being two hosts, both with minimal redundancy achieved using two different codons and a pair of parasites specializing on these hosts.

To investigate the coevolutionary dynamics of two pairs of hosts and parasites, we need to consider four different traits. In line with the previous section, we have chosen the parameter $d_{1}$ and $d_{2}$, which denote the binding rates between codon and anticodon for hosts 1 and 2 , and $\gamma_{1}$ and $\gamma_{2}$ which represent the codon abundances of parasites 1 and 2. Let the densities of host $i$ be given by $x_{i}$ and the density

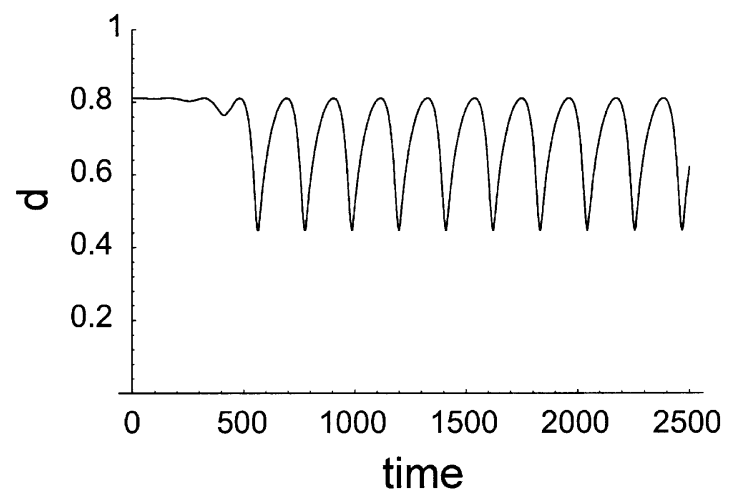

FIG. 4. The redundancy quantified over time illustrating red queen dynamics of monomorphic host and parasite populations. Parameters as in Fig. 2.

of parasite $i$ by $y_{i}$. The resident dynamics of the four host and parasites is given by

$$
\begin{aligned}
& \dot{x}_{1}=x_{1}\left(r-y_{1} q_{11}-y_{2} q_{12}\right), \\
& \dot{x}_{2}=x_{2}\left(r-y_{1} q_{21}-y_{2} q_{22}\right), \\
& \dot{y}_{1}=y_{1}\left(x_{1} q_{11}+x_{2} q_{21}-\delta\right), \\
& \dot{y}_{2}=y_{2}\left(x_{1} q_{12}+x_{2} q_{22}-\delta\right)
\end{aligned}
$$

with $q_{i j}=q\left(d_{i}, \gamma_{j}\right)$. The derivation of the evolutionary dynamics, although tedious, is similar to the derivation in the previous section (see Appendix B). Simulations show that the evolutionary cycling disappears when the host and parasite populations are dimorphic (Fig. 5). Whereas in the monomorphic case the redundancy fluctuated, here the system evolves towards minimal redundancy. In the evolutionary equilibrium each host uses a single opposing anticodon.

Once the hosts have evolved to minimal redundancy a third host type with maximal redundancy can appear. In the present model this host can never invade the system as it will be attacked by both parasites. If this host derived an advantage from having more than one anticodon available, for instance through an increased $r$ as a result of a buffering, it would be possible for it to invade and replace the two incumbent non-redundant hosts. In this way redundancy might evolve incrementally. The 

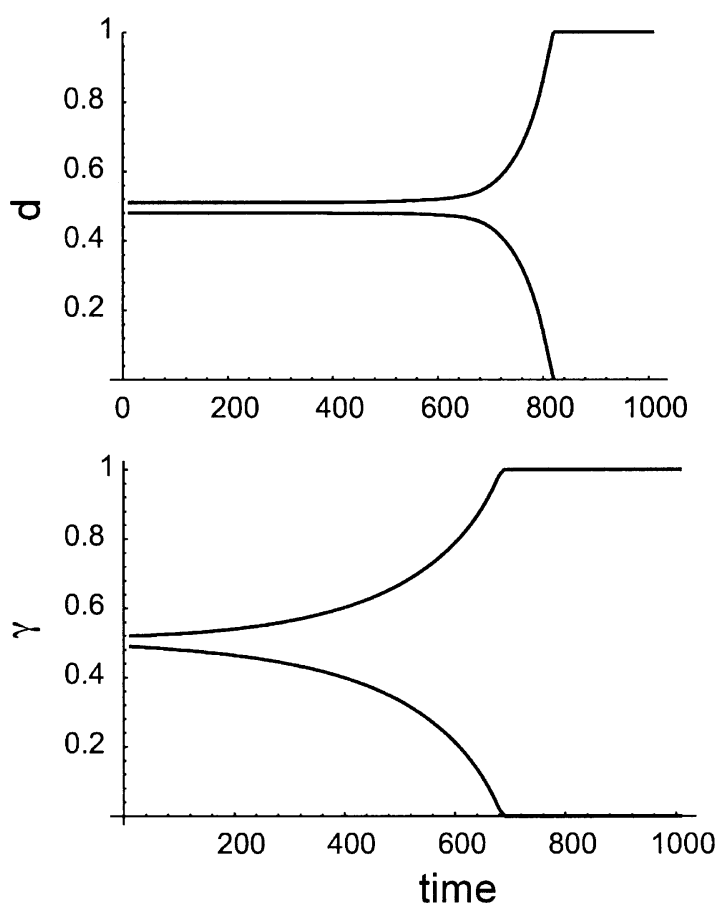

FIG. 5. The dynamics of dimorphic host and parasite populations. Both parasites $(\gamma$ parameter) and host $(d$ parameter) evolve towards minimal redundancy with nonoverlapping codon usage. Populations are initially homogeneous. Over the course of evolution the populations diverge into two-specialized solutions. Parameters as in Fig. 2.

detailed modeling and analysis of the evolutionary dynamics lies beyond the scope of this paper.

\section{Adaptive Modification of tRNA Base Composition}

The models illustrate how changes in anticodon redundancy and degeneracy are expected to modify parasite replication rates, and how parasite-host population dynamics feeds-back to influence translation. In biological systems these changes could be achieved in one of two ways: (1) changing the abundance and anticodon usage of all tRNAs in the germ line or (2) facultatively modifying individual tRNAs in infected cells or cellular levels of tRNA expression. The first solution is problematic as it would interfere with the translation of host genes, and presumably, could only occur at a fraction of the rate of change of parasite codon usage. The second strategy is preferable, as it could target individual cells and operate over very short-onto- genetic - time-scales. In this section, we provide evidence for molecular mechanisms that modify redundancy within cells by modifying binding specificity.

Transfer RNAs possess a large number of modified bases. These are chemical modifications of one of the four standard RNA bases, A, G, C and U. Modification can be modest, in which case a single methyl group is added to a base, or more dramatic, in which the purine ring is restructured. The result of modification is to change the binding affinity of the anticodon for the codon, thereby increasing or reducing adherence to canonical Watson-Crick basepairing rules. Furthermore, gene expression of each tRNA can be modified when a gene requires a minimum concentration of cognate tRNAs in order to be expressed. Consider the following examples:

(i) In papillomavirus, codon bias or modification has been observed to suppress the production of capsid protein. Upregulating tRNA expression can restore this protein (Zhou et al., 1999).

(ii) In E. coli uridine in the wobble position of tRNAGlu and tRNALys is modified to reduce the possibility of misreading near-cognate codons and reduces the range of cognate codon readings. Modification causes GAG to be translated more slowly and GAA more quickly than the average codon (Kruger et al., 1998).

(iii) Modification of tRNA bases can alter the incidence of retroviral ribosomal frameshift mutations. A modified quinine in the wobble position increases frameshifting at one frameshift site codon and reduces frameshifting at another site (Carlson et al., 1999).

(iv) In the starfish mitochondrial genome there is no tRNA anticodon able to translate all four serine codons. This deficiency is overcome by having a 7-methylguanosine at the wobble position, expanding the isoaccepting class such that the tRNA can form bases with all four nucleotides through reduced specificity (Matsuyama et al., 1998).

(v) Structural studies of tRNA with a queuosine-modified tRNAasp show that this modifies base pairing. Unmodified tRNAasp form stable association with GAC and unstable association 
with GAU. The modified form exhibits no bias for either of these codons and is thought to regulate protein synthesis under different codon biases (Morris et al., 1999).

(vi) Wild-type tRNALeu(UUR) found in mitochondria possess a modified base at the wobble position. Mutants possess an unmodified base, and this is associated with the muscle wasting MELAS disease phenotype. It is thought that mutants lacking the modification are unable to decode their cognate codons efficiently (Wright, 1990a,b).

Thus, widespread modification provides an facultative means of increasing variability in host translation machinery, without having to change genetically tRNA copy numbers or codon usage. Infected cells undergo anticodon modification handicapping the highly expressed translational parasite, without impairing significantly, translation in uninfected cells.

\section{Correlations in Parasite-Host Codon Usage}

In previous sections, we introduced models for thinking about translation kinetics and evolutionary dynamics. We then provided some evidence for modification of the anticodon leading to short-term changes in anticodon specificity. We now summarize studies in which codon bias has had a demonstrable effect on the rate of translation of parasite genes.

Within E. coli rare codons have been shown to be associated with reduced protein levels (Makrides, 1996; Hernan et al., 1992). In one study, the gene encoding human alpha-1-antitrypsin (A1AT), was cloned and expressed as a fulllength, non-fusion gene product in $E$. coli. Mutagenesis in vitro to the $5^{\prime}$-terminal region of the gene aimed at improving codon usage in accordance with its host, increased expression 10-20-fold (Sutiphong et al., 1987).

Codon biases can also be used to regulate gene expression within a single genome. Translational pauses within the coat protein of the RNA bacteriophage MS2 have been located on the basis of a distribution plot of rare codons and RNA secondary structure. Discontinuous translation rates resulting from these patches of rare codons are thought to allow for optimal folding of the polypeptide (Guisez et al., 1993).
The Epstein-Barr virus (EBV) can exist either in a latent state or as a productive infection. A small set of genes are expressed exclusively during latency, and a larger set exclusively during production. There is a significant difference in the codon usage between these two sets of genes. In particular, codon usage among latent genes are markedly different from those of the host, whereas codon usage of the productive genes are more similar to those of the host (Karlin et al., 1990). In one case, the codon difference is assumed to minimize the deleterious impact of expression during latency, and in the other, to maximize the rate of translation.

Codon usage of gene sequences derived from Autographa californica nuclear polyhedrosis virus (AcNPV) were compared with that of firefly luciferase (luc) and the beta subunit of human chorionic gonadotropin (beta hCG) expressed at varying levels in a baculovirus system. The most highly expressed gene of the set was the luc gene that showed a codon usage similar to AcNPV genes and a similar $\mathrm{G} / \mathrm{C}$ usage at wobble positions (Ranjan \& Hasnain, 1995).

Using a maximum-likelihood statistical method, base composition and codon usage bias have been shown to covary with arthropod association in the genus Flavivirus (Jenkins et al., 2001). Flaviviruses are transmitted by mosquitoes, ticks, and between vertebrate hosts. Viruses associated with ticks have a significantly lower $\mathrm{G}+\mathrm{C}$ content than non-vector-borne flaviviruses. Mosquito-borne viruses have an intermediate $G+C$ content which is not significantly different from those of the other two groups. Furthermore, biases in codon usage that are independent from base composition are detected in all flaviviruses, however, these do not covary with arthropod association.

Human papillomavirus type 16 (HPV-16) is a major cause of cervical neoplasia, but only a minority of HPV-16 infections result in cancer. An investigation of whether cervical neoplasia is associated with infection with HPV-16 intratypic variants was undertaken by using RFLP analyses in a study of $100 \mathrm{HPV}-16$ DNA-positive women with or without neoplasia. The associations between HPV-16 E5 RFLP variants and neoplasia could not be attributed to differences 
in amino acid sequences, whereas a strong correlation was observed in codon usage (Bible et al., 2000). It seems therefore that the translational advantage gained through codon usage can make the difference between cancerous and non-cancerous cell lineages.

To test the possibility that papillomavirus (PV) late gene codon composition determines the efficiency of PV late gene expression in some cell types, synthetic bovine papillomavirus type 1 (BPV1) late genes were constructed with codon composition modified to resemble the typical mammalian gene. Expression of these genes from a strong promoter in Cos-1 cells was compared with expression of wild-type BPV1 late genes from the same promoter. Both unmodified and modified PV late genes were transcribed in Cos-1 cells, but only the codonmodified genes were translated. Codon composition thus limits BPV1 late gene translation in Cos- 1 cells, and this limitation can be overcome by modification of the codon composition of the genes or by provision of excess tRNA (Zhou et al., 1999). This study is particularly intriguing as it demonstrates that inapropriate codon usage can lead to a complete shut-off of translation.

Selective codon usage appears to account for a substantial fraction of the inefficiency of viral protein synthesis. Re-engineering the coding sequence of a model protein (Thy-1) with the most prevalent HIV-1 codons significantly impairs Thy-1 expression, whereas altering the coding sequence of the jellyfish green fluorescent protein gene to conform to the favored codons of highly expressed human proteins results in a substantial increase in expression efficiency (Haas et al., 1996).

A requirement of human immunodeficiency virus (HIV) vaccines is that they induce potent and durable cellular immune responses. In an effort to mount a strong response against an HIV-specific epitope, the highly conserved protein Gag was selected for modification. Viral codons were changed to conform to the codon usage of highly expressed human genes, and residual inhibitory sequences were removed. The resulting modified gag gene showed increases in $\mathrm{p} 55(\mathrm{Gag})$ protein expression to levels that ranged from 322- to 966 -fold greater than that for the native gene after transient expression of 293 cells (Zur Megede et al., 2000).

A synthetic human immunodeficiency virus type 1 gp120 sequence was generated in which most wild-type codons were replaced with codons from highly expressed human genes (syngp120). In vitro expression of syngp120 was shown to be considerably increased in comparison to that of the respective wild-type sequence (Andre et al., 1998).

The foregoing examples all serve to illustrate how tight coupling between the host translational machinery and virus codon usage can have a significant impact on the translation rate of the parasite genome, and indirectly on host fitness. It is worth emphasizing at this point that codon bias can result from any number of processes including mutational bias (Bulmer, 1991), the demands made by DNA folding and stability (Karlin \& Mrazek, 1996) and translation selection (Sharp et al., 1995). We have concentrated on the latter because we are interested in the influence of parasitism on the genetic translation machinery and the diversity of the genetic anticode, not because we believe that translation selection is the dominant cause of codon bias.

\section{Summary and Conclusions}

The mechanisms that organisms employ to translate proteins remain highly conserved across taxa. However, at the level of the anticodon-codon recognition, there is a great deal of variation both within and between species. This variation is reflected in codon usage, tRNA copy number, and anticodon redundancy, and degeneracy. Whereas the genetic code is highly conserved, the genetic anticode is diverse. In this paper, degeneracy and redundancy in the translational machinery of the genetic code have been explored in relation to infection with parasites utilizing host protein-synthesis pathways. We have suggested that these features of the translational machinery can be understood, in part, as adaptations to reduce the costs of infection. The translational apparatus acts as a "primitive" mechanism of resistance. Assuming that codon usage influences parasite replication rate in an asexual host, a monomorphic host 
population (one where all hosts adopt the same translational strategy) continually modifies its tRNAs so as to reduce parasite translation efficiency. Mechanisms which facilitate epigenetic modification of the anticodon, modifying codon-anticodon binding specificity, are likely to have evolved to bring the rate of host evolution in line with the rapid genetic evolution of parasites.

When populations contain more than one host type (hosts vary in their anticodon redundancy and degeneracy), then each host and parasite pair evolve towards low levels of redundancy whereas the population as a whole becomes polymorphic in codon usage. Thus, each parasite becomes a translational specialist on a unique host tRNA background.

By now evolutionary biologists are familiar with the hypothesis that parasites exert a strong selection pressure on the evolution of sex (Hamilton et al., 1990). This theory does not address the fine structure of the genetic apparatus, but deals with reductive cell division, recombination and the cost of males. We have found that in both monomorphic and polymorphic asexual populations, parasites can promote the diversity of the translational process itself. In terms of genetic organization, there appears to be "plenty of room at the bottom" for parasite-host theories.

D.C.K. thanks the Alfred P Sloan Foundation, The Ambrose Monell Foundation, The Florence Gould Foundation, the J. Seward Johnson Trust and the NSF. We thank the three anonymous referees for their constructive comments and suggestions.

\section{REFERENCES}

Anderson \& Kurland, C. G. (1998). Reductive evolution of resident genomes. Trends Microbiol. 6, 263-268.

Andre, S., Seed, B., Eberle, J., Schraut, W., Bultmann, A. \& HAAS, J. (1998). Increased immune response elicited by DNA vaccination with a synthetic gp120 sequence with optimized codon usage. J. Virol. 72, 1497-1503.

Bennetzen, J. L. \& Hall, B. D. (1982). Codon selection in yeast. J. Biol. Chem. 257, 3026-3031.

Bible, J. M., Mant, C., Best, J. M., Kell, B., Starkey, W. G., Shanti Raju, K., Seed, P., Biswas, C., Muir, P., Banatvala, J. E. \& Cason, J. (2000). Cervical lesions are associated with human papillomavirus type 16 intratypic variants that have high transcriptional activity and increased usage of common mammalian codons. J. Gen. Virol. 81(6), 1517-1527.
Bulmer, M. (1991). The selection-mutation-drift theory of synonymous codon usage. Genetics 129, 897-907.

Carlson, B. A., Kwon, S. Y., Chamorro, M., Oroszlan, S., Hatfield, D. L. \& Lee, B. J. (1999). Transfer RNA modification status influences retroviral ribosomal frameshifting. Virology 255, 2-8.

Dieckmann, U. \& Law, R. (1996). The dynamical theory of coevolution: a derivation from stochastic ecological processes. J. Math. Biol. 34, 579-612.

Dong, H., Nilsson, L. \& Kurland, C. G. (1995). Gratuitous overexpression of genes in Escherichia coli leads to growth inhibition and ribosome destruction. J. Bacteriol. 177, 1497-1504.

Fitch, W. M., Bush, R. M., Bender, C. A. \& Cox, N. J. (1997). Long term trends in the evolution of H(3) HA1 human influenza type A. Proc. Natl Acad. Sci. U.S.A. 94, $7712-7718$.

FrANK, S. A. (1994). Recognition and polymorphism in host-parasite genetics. Philos. Trans. R. Soc. Lond. B Biol. Sci. 346, 283-293.

Freeland, S. J., Hurst, L. D. (1998). The genetic code is one in a million. J. Mol. Evol. 47, 238-248.

Guisez, Y., Robbens, J., Remaut, E. \& Fiers, W. (1993). Folding of the MS2 coat protein in Escherichia coli is modulated by translational pauses resulting from mRNA secondary structure and codon usage: a hypothesis. J. theor. Biol. 162, 243-252.

Haas, J., Park, E. C. \& Seed, B. (1996). Codon usage limitation in the expression of HIV-1 envelope glycoprotein. Curr. Biol. 6, 315-324.

Haig, D. \& Hurst, L. D. (1991). A quantitative measure of error minimization in the genetic code. J. Mol. Evol. 33, $412-417$.

Hamilton, W. D., Axelrod, R. \& Tanese, R. (1990). Sexual selection as an adaptation to resist parasites (a review). Proc. Natl Acad. Sci. U.S.A. 87, 3566-3573.

Hernan, R. A., Hui, H. L., Andracki, M. E., Noble, R. W., Sligar, S. G., Walder, J. A. \& Walder, R. Y. (1992). Human hemoglobin expression in Escherichia coli: importance of optimal codon usage. Biochemistry 31, 8619-8628.

Jenkins, G. M., Pagel, M., Gould, E. A., Zanotto, P. M. \& Holmes, E. C. (2001). Evolution of base composition and codon usage bias in the genus flavivirus. J. Mol. Evol. 52, 383-390.

Karlin, S. \& MrazeK, J. (1996). What drives codon choices in human genes? J. Mol. Biol. 262, 459-472.

Karlin, S., Blaisdell, B. E. \& Schachtel, G. A. (1990). Contrasts in codon usage of latent versus productive genes of Epstein- Barr virus: data and hypotheses. J. Virol 64, 4264-4273.

Knight, R. D., Freeland, S. J. \& Landweber, L. F. (1999). Selection, history and chemistry: the three faces of the genetic code [see comments]. Trends Biochem. Sci. 24, 241-247.

KraKaUer, D. C. (2002). Evolutionary principles of genomic compression. Comm. theor. Biol.

Kruger, M. K., Pederson, S., Hagervall, T. G. \& SorESON, M. A. (1998). The modification of the wobble base of tRNAGlu modulates the translation rate of glutamic acid codon in vivo. J. Mol. Evol. 284, 621-631.

KuRland, C. G. (1991). Codon bias and gene expression. FEBS Lett. 285, 165-169. 
Lammertyn, E., Van Mellaert, L., Bijnens, A. P., Joris, B. \& AnNe, J. (1996). Codon adjustment to maximize heterologous gene expression in Streptomyces lividans can lead to decreased mRNA stability and protein yield. Mol. Gen. Genet. 250, 223-229.

LANDE, R. (1979). Quantitative genetics analysis of multivariate evolution, applies to brain: body size allometry. Evolution 33, 402-416.

Makrides, S. C. (1996). Strategies for achieving high-level expression of genes in Escherichia coli. Microbiol. Rev. 60, 512-538.

Matsuyama, S., Ueda, T., Crain, P. F., McCloskey, J. A. \& Watanabe, K. (1998). A novel wobble rule found in starfish mitochondria. Presence of 7-methylguanosine at the anticodon wobble position expands decoding capability of tRNA. J. Biol. Chem. 273, 3363-3368.

Metz, J. A. J., Nisbet, R. M. \& Geritz, S. A. H. (1992). How should we define fitness for general ecological scenarios. Trends Ecol. Evol. 7, 198-202.

Morris, R. C., Brown, K. G. \& Elliott, M. S. (1999) The effect of queuosine on tRNA structure and function. J. Biomol. Struct. Dynamics 16, 757-774.

Mylius, S. D. \& Diekmann, O. (1995). On evolutionarily stable life histories, optimization and the need to be specific about density dependence. Oikos 74, 218-224.

Percudani, R., Pavesi, A. \& Ottonello, S. (1997). Transfer RNA gene redundancy and translational selection in Saccharomyces cerevisiae. J. Mol. Biol. 268, 322-330.

Ranjan, A. \& Hasnain, S. E. (1995). Codon usage in the prototype baculovirus-Autographa californica nuclear polyhedrosis virus. Ind. J. Biochem. Biophys. 32, 424- 428.

Sharp, P. M., Stenico, M., Peden, J. F. \& Lloyd, A. T. (1993). Codon usage: mutational bias, translational selection, or both? Biochem. Soc. Trans. 21, 835-841.

Sharp, P. M., Averof, M., Lloyd, A. T., Matassi, G. \& Peden, J. F. (1995). DNA sequence evolution: the sounds of silence. Philos. Trans. R. Soc. Lond. B Biol. Sci. 349, 241-247.

Sutiphong, J., Johansen, H., Sathe, G., Rosenberg, G. S. \& Shatzman, A. (1987). Selection of mutations that increase alpha 1-antitrypsin gene expression in Escherichia coli. Mol. Biol. Med. 4, 307-322.

van BaAlen, M. \& Jansen, V. A. A. (2001). Dangerous liaison: the ecology of private interest and common good. Oikos 95, 211-224.

VAn Valen, L. M. (1973). A new evolutionary law. Evol. Theory 1, 1-30.

Wright, F. (1990a). The 'effective number of codons' used in a gene. Gene 87, 23-29.

Wright, F. (1990b). Defect in the modification of anticodon wobble base of mutant mitochondrial tRNAs in MELAS mitochondrial encephalomyopathy 87, 23-29.

XIA, X. (1998). How optimized is the translational machinery in Escherichia coli, Salmonella typhimurium and Saccharomyces cerevisiae? Genetics 149, 37-44.

Zhou, J., Liu, W. J., Peng, S. W., Sun, X. Y. \& Frazer, I. (1999). Papillomavirus capsid protein expression level depends on the match between codon usage and tRNA availability. J. Virol. 73, 4972-4982.

Zhou, J., Liu, W. J., Peng, S. W., Sun, X. Y. \& Frazer, I. (1999). Papillomavirus capsid protein expression level depends on the match between codon usage and tRNA availability. J. Virol. 73, 4972-4982. zur Megede, J., Chen, M. C., Doe, B., Schaefer, M., Greer, C. E., Selby, M., Otten, G. R. \& Barnett, S. W. (2000). Increased expression and immunogenicity of sequence-modified human immunodeficiency virus type 1 gag gene. J. Virol. 74, 2628-2635.

\section{APPENDIX A}

In this appendix, we formulate a general description of the translation process. We assume that there are $m$ different codons, $m$ anticodons, and $n$ amino acids. The $m \times 1$ vector c specifies the abundances of each of the $m$ codons in the RNA strain. The process of translation involves matching these codons with tRNA anticodons. The abundance of anticodon $i$ in the cell is given by elements $v_{i}$ of the anticodon vector $\mathbf{v}$. The binding rate of anticodon $i$ with codon $j$ is given by element $w_{i j}$ of the binding matrix $\mathbf{W}$.

The total rate of binding to codon $j$ is given by $\sum_{i}^{m} v_{i} w_{i j}$, hence the average time it takes for a codon to be matched is given by

$$
f_{j}=\left(\sum_{i}^{m} v_{i} w_{i j}\right)^{-1} .
$$

It also follows that codon $j$ is matched with anticodon $i$ with probability

$$
u_{i j}=v_{i} w_{i j} f_{j}
$$

Because each codon will be matched with an anticodon, we have $\sum_{i=1}^{m} u_{i j}=1$. The $m \times m$ matrix $\mathbf{U}$ has the probabilities $u_{i j}$ as elements. Each tRNA anticodon is associated with an amino acid. The association between anticodon and amino acid is described by the $n \times m$ binary (i.e. containing only zeros and ones) matrix $\mathbf{A}$. The elements of $\mathbf{A}$ are denoted $a_{i j}$. Each column of $\mathbf{A}$ consist of $n-1$ zeros and only a single one as there is a unique association between tRNA and amino acid. In case there is degeneracy rows of $\mathbf{A}$ can contain many ones. Binary $\mathbf{U}$ matrices are degenerate with no redundancy. Matrices in which any element $u_{i j}<1$, have redundancy in the $i$ th anticodon. The abundance in amino acids after translation is given by AUc.

The total translation time is given by the sum of the time it takes to initiate the translation process, $\varepsilon$ (binding of the message to the 
ribosome), and the total time to match all codons

$$
\tau=\varepsilon+\sum_{j}^{m}\left(c_{j} f_{j}\right) .
$$

Assuming that the extension of the protein is the rate-limiting step, and not the removal of improperly bound anticodons, the rate of translation is given by $q=1 / \tau$.

We distinguish between parasite and host codon usage. Let the $m \times 1$ vector $\mathbf{c}_{p}$ contain the abundances of codons in the parasite genome. The first step in translation is the transcription into mRNA, which is a possible source of error. Another source of error is mutation during replication. The rate of mutation of parasite codons during replication is determined by the matrix B where elements $b_{i j}$ specify the probability of codon $i$ mutating into codon $j$. Thus, the effective number of codons of type $i$ is determined by the product of the mutation matrix and the codon abundance vector,

$$
\pi=\mathbf{B c}_{p} .
$$

The total time needed for translation of a parasite $l$ in a host $k$ is given by $\tau_{l k}$. Within a host cell, $\tau_{l k}$ is derived from the degree of complementarity between the parasite and host genomes and is derived form the mean time for translation of all parasite codons:

$$
\tau_{l k}=\varepsilon+\sum_{j}^{m}\left(\pi_{j} f_{j}\right) .
$$

The rate of translation of parasite $l$ in a host $k$ is $q_{l k}=1 / \tau_{l k}$ A parasite is incapable of infecting a host when $q_{l k}=0$. This will result when $\sum_{i} f_{i} \pi_{i}$ tends to infinity, i.e. when the parasite has a set of codons which find no match in the host's set of anticodons.

\section{APPENDIX B}

To derive the evolutionary dynamics in a system of two hosts and two parasites, we first observe that the equilibrium values of system are given by:

$$
\hat{x}_{1}=\frac{\delta\left(q\left(d_{2}, \gamma_{2}\right)-q\left(d_{2}, \gamma_{1}\right)\right)}{q\left(d_{1}, \gamma_{1}\right) q\left(d_{2}, \gamma_{2}\right)-q\left(d_{2}, \gamma_{1}\right) q\left(d_{1}, \gamma_{2}\right)},
$$

$$
\begin{aligned}
& \hat{x}_{2}=\frac{\delta\left(q\left(d_{1}, \gamma_{1}\right)-q\left(d_{1}, \gamma_{2}\right)\right)}{q\left(d_{1}, \gamma_{1}\right) q\left(d_{2}, \gamma_{2}\right)-q\left(d_{2}, \gamma_{1}\right) q\left(d_{1}, \gamma_{2}\right)}, \\
& \hat{y}_{1}=\frac{r\left(q\left(d_{2}, \gamma_{2}\right)-q\left(d_{1}, \gamma_{2}\right)\right)}{q\left(d_{1}, \gamma_{1}\right) q\left(d_{2}, \gamma_{2}\right)-q\left(d_{2}, \gamma_{1}\right) q\left(d_{1}, \gamma_{2}\right)}, \\
& \hat{y}_{2}=\frac{r\left(q\left(d_{1}, \gamma_{1}\right)-q\left(d_{2}, \gamma_{1}\right)\right)}{q\left(d_{1}, \gamma_{1}\right) q\left(d_{2}, \gamma_{2}\right)-q\left(d_{2}, \gamma_{1}\right) q\left(d_{1}, \gamma_{2}\right)}
\end{aligned}
$$

and that also in this system, the long-term averages of the densities converge to the equilibrium values.

The dynamics of rare mutant hosts $x_{1}^{*}$ and $x_{2}^{*}$ and parasites $y_{1}^{*}$ and $y_{2}^{*}$ with respective traits $d_{1}^{*}$, $d_{2}^{*}, \gamma_{1}^{*}$ and $\gamma_{2}^{*}$ are given by:

$$
\begin{aligned}
& \dot{x}_{1}^{*}=x_{1}^{*}\left(r-\hat{y}_{1} q\left(d_{1}^{*}, \gamma_{1}\right)-\hat{y}_{2} q\left(d_{1}^{*}, \gamma_{2}\right),\right. \\
& \dot{x}_{2}^{*}=x_{2}^{*}\left(r-\hat{y}_{1} q\left(d_{2}^{*}, \gamma_{1}\right)-\hat{y}_{2} q\left(d_{2}^{*}, \gamma_{2}\right),\right. \\
& \dot{y}_{1}^{*}=y_{1}^{*}\left(\hat{x}_{1} q\left(d_{1}, \gamma_{1}^{*}\right)+\hat{x}_{2} q\left(d_{2}, \gamma_{1}^{*}\right)-\delta\right), \\
& \dot{y}_{2}^{*}=y_{2}^{*}\left(\hat{x}_{1} q\left(d_{1}, \gamma_{2}^{*}\right)+\hat{x}_{2} q\left(d_{2}, \gamma_{2}^{*}\right)-\delta\right)
\end{aligned}
$$

Following the same reasoning as before, we can derive the evolutionary dynamics as a system of four coupled ordinary differential equations of the form:

$$
\begin{gathered}
\dot{d}_{1}=-\mu_{d} \hat{x}_{1}\left(\left.\hat{y}_{1} \frac{\partial q\left(d, \gamma_{1}\right)}{\partial d}\right|_{d=d_{1}}+\left.\hat{y}_{2} \frac{\partial q\left(d, \gamma_{2}\right)}{\partial d}\right|_{d=d_{1}}\right), \\
\dot{d}_{2}=-\mu_{d} \hat{x}_{2}\left(\left.\hat{y}_{1} \frac{\partial q\left(d, \gamma_{1}\right)}{\partial d}\right|_{d=d_{2}}+\left.\hat{y}_{2} \frac{\partial q\left(d, \gamma_{2}\right)}{\partial d}\right|_{d=d_{2}}\right), \\
\dot{\gamma}_{1}=\mu_{\gamma} \hat{y}_{1}\left(\left.\hat{x}_{1} \frac{\partial q\left(d_{1}, \gamma\right)}{\partial \gamma}\right|_{\gamma=\gamma_{1}}+\left.\hat{x}_{2} \frac{\partial q\left(d_{2}, \gamma\right)}{\partial \gamma}\right|_{\gamma=\gamma_{1}}\right), \\
\dot{\gamma}_{2}=\mu_{\gamma} \hat{y}_{2}\left(\left.\hat{x}_{1} \frac{\partial q\left(d_{1}, \gamma\right)}{\partial \gamma}\right|_{\gamma=\gamma_{2}}+\left.\hat{x}_{2} \frac{\partial q\left(d_{2}, \gamma\right)}{\partial \gamma}\right|_{\gamma=\gamma_{2}}\right) .
\end{gathered}
$$

The values of all evolutionary parameters are restricted to the unit interval. It is easy to show that $d_{1}=d_{2}=\gamma_{1}=\gamma_{2}=1 / 2$ is an equilibrium of this and that this equilibrium is unstable. For further results, we numerically integrated this system of ODE's. 